\title{
An oscillatory component of the H-reflex
}

\author{
JOH N W C A T ON AND RICHARD R RUED \\ From the Department of Psychiatry, The University of Chicago, Chicago, Illinois
}

SUMMARY H-reflex recovery curves studied in 57 normal volunteers could be divided into three groups: one with sinusoidal properties having one or more periods, one having primarily linear or exponential properties, and a third, indeterminate group.

Since the original publication by Hoffmann, ${ }^{1}$ numerous reports have appeared describing variout features of the H-reflex in normal and pathological subjects. Studies by Magladery and McDougal $^{2}$ and Paillard ${ }^{3}$ stimulated interest in the relationship of the $\mathrm{H}$-reflex recovery curve to neuropathological states such as Parkinson's disease, stroke, cord trauma, and various other neurological diseases. ${ }^{4-6}$ In reviewing this literature, we have noted that the configuration of the $\mathrm{H}$-reflex recovery curve is invariably described as having an initial period of facilitation (8-12 ms delay), best shown at near threshold stimulation, a "secondary facilitation" at $100-300 \mathrm{~ms}$ delay, followed by a trough, and then gradual return to $100 \%$ excitability in 5-10 seconds. During the course of our studies on the determinates of recovery curve parameters, we have found evidence that the H-reflex recovery curve is more complex than has been reported previously.

\section{Methods}

Fifty-seven volunteer adults were studied, ranging in age from 18 to 44 years. All were screened for the presence of neurological disease and for a family history of mental or neurological abnormalities. Twin square-wave pulses of $1 \mathrm{~ms}$ duration were delivered to the posterior tibial nerve via a bipolar surface stimulating electrode placed in the popliteal fossa. Recording was achieved with metal disc electrodes placed over the tendon and belly of the soleus muscle. Responses were amplified with a Grass P511 pre-amplifier and were displayed on a Tektronix storage oscilloscope D13 or processed by a PDP-8 computer. Stimulus strength

Address for reprint requests: Dr JW Crayton, Department of Psychiatry, University of Chicago, 950 East 59th St, Chicago, III, 60637, USA

Accepted 30 July 1980 was adjusted to achieve a maximal $\mathrm{H}$-response. In most cases, S1-S2 delay intervals used were 30, $50,70,90,100,150,200,300,400,600,800,1000$, 2000 , and $5000 \mathrm{~ms}$. Five determinations per delay were taken for these data and curves plotted from mean $\mathrm{H} 2 / \mathrm{H} 1$ values at each delay. In two additional studies, recovery was sampled at equidistant 50 and $100 \mathrm{~ms}$ intervals between 50 and $2000 \mathrm{~ms}$. Three determinations per delay were taken for these data. While no direct determination of variability in stimulus strength was made, stimuli were presented in a random sequence of S1-S2 delay intervals, so any random fluctuations in stimulus intensity would be unlikely sources of recovery variations.

\section{Results}

When studied at a stimulus strength eliciting a maximal $\mathrm{H}$-response, the initial facilitation at 8-12 ms was not seen. Secondary facilitation ( $\mathrm{Hx})$ between $100-300 \mathrm{~ms}$ was reached at an average value of $66.3 \pm 29.9 \%$, which is similar to that reported by others. The presence or absence of a small $M$ response was not significantly correlated with recovery curve parameters. On inspection of the individual recovery curve graphs it seemed plausible that the large standard deviation for the group as a whole might be due to sub-groups of H-reflex curve types. Three predominant categories of curves could be distinguished. One group of curves appeared to have sinusoidal properties, marked by one, two, or more periods (fig 1A, 1B). Higher order facilitatory peaks are also discernible in the curves illustrated in fig 2 , where small uniform $(50 \mathrm{~ms})$ increments in S1-S2 delay increase curve resolution. A second group of curves was characterised by monotonic increasing functions, that is, linear or exponential behaviour (fig 1C, 1D). The last group consisted of curves that could 


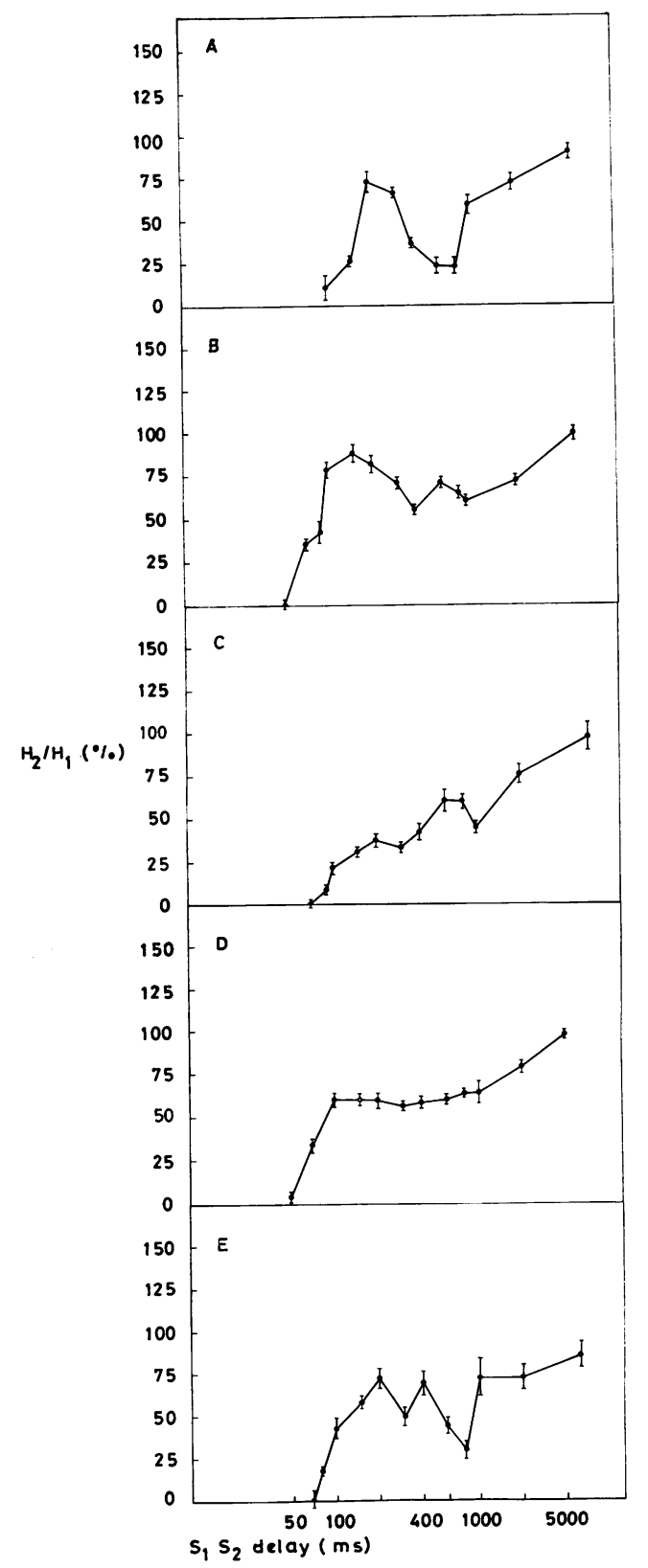

Fig 1 H-reflex recovery curves. Each curve represents recovery in a single individual; each point is the mean of five determinations. A Curve with a single facilitatory peak, group I curve. B Two distinct facilitatory peaks, group I curve. C "Linear" curve, group II curve. D Exponential curve, group II curve. E Uncertain curve, group III curve. Vertical bars indicate standard error of the mean. not clearly be classified into either of the above categories (fig 1E).

Classification was made by an independent rater who was given a description of the curve types and asked to classify each curve accordingly. Curves exhibiting sinusoidal properties comprised $54.4 \%$ of the total $(31 / 57)$, of which $83.9 \%(26 /$ 31) gave evidence of two peaks prior to $1000 \mathrm{~ms}$ delay. Twenty-one curves $(36.8 \%)$ were classified as monotonically increasing. The remainder of the curves $(5 / 57$, or $8.8 \%)$ were categorised as uncertain. There was no relationship between age or sex and curve types. Given these curve types, one might hope to predict them from some feature of the recovery curve. If each curve is seen as being indicative of the response of a second order non-linear servomechanism, oscillatory curves might be construed as under-damped curves and exponential or linear curves as over-damped curves. $^{7}$ Thus, the value of recovery at secondary facilitation ( $\mathbf{H x})$ would tend to be higher for under-damped curves and therefore higher for the sinusoidal category of curve type. Likewise, $\mathrm{Hx}$ would tend to be lower for over-damped curves and therefore lower for the monotonically increasing category of curve type. To test this hypothesis, a hierarchical cluster analysis was performed on Hx. The particular technique used was the nearest neighbour method by which groups are generated according to the distance between single indi-

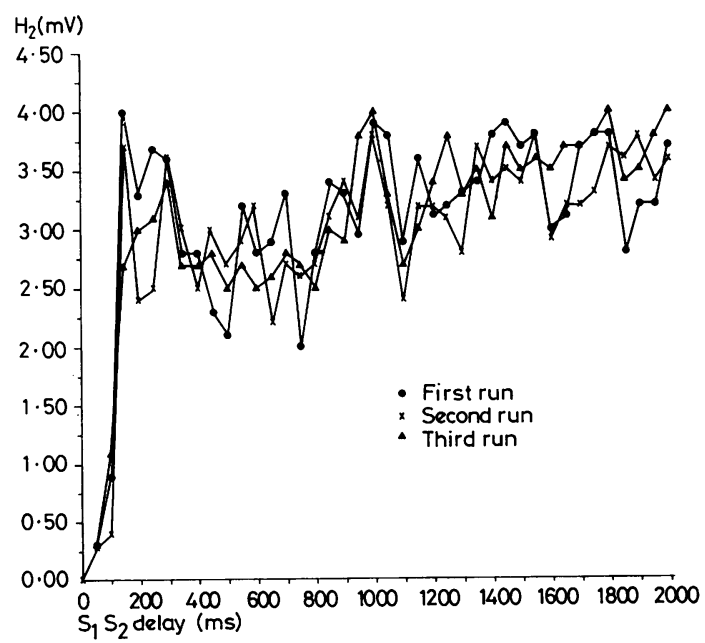

Fig $2 H$-reflex recovery curve. Equi-interval method. S1-S2 increments of $50 \mathrm{~ms}$. Each curve is a run and each point a single determination. The presence of peaks at 150 and 1000 ms delay are clearly seen. Other smaller peaks may also be present. 
viduals, the smallest distance being fused to the group. A detailed account of this technique is given by Johnson. ${ }^{8}$ Each element is agglomerated until some specified minimum number of groups is achieved. The number of groups selected of $\mathbf{H x}$ was three to complement the three curve types. The descriptive statistics for each cluster are summarised in table 1.

If the hypothesis that the value of $\mathrm{Hx}$ is correlated with curve type holds, then a cross tabulation of $\mathrm{Hx}$-cluster with curve type should demonstrate that curves with sinusoidal properties will occur predominately in cluster one (high $\mathbf{H x}$ ) to some extent in cluster two (medium $\mathbf{H x}$ ), and rarely in cluster three (low Hx). Likewise, curves with monotonically increasing properties should occur predominately in cluster three (low $\mathrm{Hx}$ ), to some extent in cluster two (medium $\mathbf{H x}$ ), and rarely in cluster one (high $\mathbf{H x}$ ). The third category of curve type should not be skewed with respect to any one category.

Table 2 summarises the results. Oscillatory curve types are contained almost exclusively (28/ 31 or $90.3 \%$ ) in the high and medium Hx clusters. Cluster one consists of $84.6 \%(11 / 13)$ oscillatory curve type, while cluster two consists of $65.4 \%$ $(17 / 26)$ and cluster three contains only $16.7 \%$ $(3 / 18)$ of that type. The low $\mathrm{Hx}$ cluster consists of $72.2 \%$ of such curves. The third group of curves is not skewed over the clusters. The chi-square

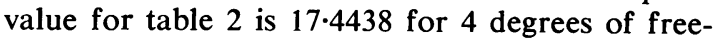
dom $(\mathrm{p}<0.001)$.

Table 1 Values for Hx in each cluster

\begin{tabular}{llll}
\hline Statistic & $\begin{array}{l}\text { Cluster one } \\
\left(\text { High } H_{x}\right)\end{array}$ & $\begin{array}{l}\text { Cluster two } \\
\left.\text { (Medium } H_{x}\right)\end{array}$ & $\begin{array}{l}\text { Cluster three } \\
\left(\text { Low } H_{x}\right)\end{array}$ \\
\hline $\begin{array}{l}\text { Mean } \\
\text { Standard }\end{array}$ & $106 \cdot 7$ & $70 \cdot 5$ & $31 \cdot 6$ \\
deviation & 12.73 & $9 \cdot 63$ & $9 \cdot 39$ \\
$\begin{array}{l}\text { Median } \\
\text { N-\% Total }\end{array}$ & 106.0 & $71 \cdot 0$ & $31 \cdot 0$ \\
\hline
\end{tabular}

Table 2 Crosstabulation of $H x$ clusters and curve groups

\begin{tabular}{|c|c|c|c|c|}
\hline \multirow[b]{2}{*}{ Clusters } & \multicolumn{3}{|l|}{ Groups } & \multirow[b]{2}{*}{ Total } \\
\hline & $\begin{array}{l}\text { One } \\
\text { (Oscillatory) }\end{array}$ & $\begin{array}{l}\text { Two } \\
\text { (Monotonic) }\end{array}$ & $\begin{array}{l}\text { Three } \\
\text { (Uncertain) }\end{array}$ & \\
\hline $\begin{array}{l}\text { One } \\
\left(\text { High } \mathbf{H}_{\mathbf{x}}\right) \\
\text { Two } \\
\left(\text { Medium } \mathbf{H}_{\mathbf{x}} \text { ) }\right. \\
\text { Three } \\
\text { (Low } \mathbf{H}_{\mathbf{x}} \text { ) } \\
\text { Total }\end{array}$ & $\begin{array}{c}11 \\
(35 \cdot 5 \%) \\
17 \\
(54 \cdot 8 \%) \\
3 \\
(9 \cdot 7 \%) \\
31 \\
(54 \cdot 4 \%)\end{array}$ & 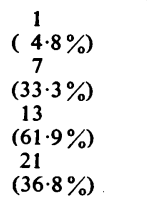 & $\begin{array}{c}1 \\
(20.0 \%) \\
2 \\
(40.0 \%) \\
2 \\
(40.0 \%) \\
5 \\
(8.8 \%)\end{array}$ & $\begin{array}{l}13 \\
(22 \cdot 8 \%) \\
26 \\
(45 \cdot 6 \%) \\
18 \\
(31 \cdot 6 \%) \\
57\end{array}$ \\
\hline
\end{tabular}

Given the high predictability of curve type from $\mathrm{Hx}$, we sought other possible associated features. Correlations were sought between S1 S2 delay at the onset of recovery, at $\mathrm{Hx}$, at $\mathrm{Hy}$ (the value of $\mathrm{H} 2 / \mathrm{H} 1 \%$ for the first trough following $\mathrm{Hx}$ ), and at $100 \%$ recovery. None of these measures approached statistical significance as predictors of curve type. It is possible that some combination of measures would be more accurate predictors of curve types and might lead to more refined delineation of curves.

\section{Discussion}

The H-reflex recovery curve consists of not just a "primary" and "secondary" facilitation. In some cases there are tertiary and higher order facilitations of diminishing amplitude as recovery becomes complete. In the case of the recovery curve illustrated in fig 2 , recovery is quite possibly not a matter of one or two but of many facilitations. A more detailed analysis of this curve and others generated with small equi-interval S1S2 delays may permit a more precise mathematical expression of the recovery function. Preliminary mathematical modelling has suggested that recovery curves can be mathematically approximated by a function that includes an exponential component. Not all individuals have clearly oscillatory recovery. In some cases recovery appears to follow an exponential function only, with little or no oscillatory behaviour. Such curves may be classified as "overdamped" (for example, fig 1c).

Oscillatory behaviour of neuro-muscular systems is common, for example, in rhythmic heartbeat and respiration. Such oscillatory movement can arise from a number of sources. Oguztoreli and Stein $^{9}$ list five sources: intrinsic muscular oscilation, load interaction oscillation, oscillations due to instabilities in neural feedback control mechanisms, oscillations contained within the CNS, and oscillation imposed by another oscillatory system. Intrinsic oscillations are not observable under normal conditions. Phenomena like "physiological" tremor $(8-12 \mathrm{~Hz})$ generally fall into the load-interaction category. ${ }^{7-10}$ Parkinsonian tremor and that of cerebellar diseases $(4-6 \mathrm{~Hz})$ probably fall into the central gene-ation category ${ }^{11}$ or the imposed category. ${ }^{12}$ The source of oscillations described here is not immediately apparent, although the most likely sources are probably oscillations in the stretch-reflex servo-loop. Other possible mechanisms to be considered are long-loop supraspinal influences, ${ }^{13}$ and Renshaw cell recurrent inhibition. ${ }^{10}$ It is interesting to note the 
probable identification of oscillatory H-reflex recovery curves in other recent studies. Hayes et al $^{14}$ comment tentatively on a $12.5 \mathrm{~Hz}$ periodicity to H-reflex recovery curves and Katz et al ${ }^{15}$ present data that could well be most accurately described by an oscillatory function (for example, their fig 4). In both cases, the oscillatory features of the recovery curve were revealed by studying the recovery curve with small $(25-100 \mathrm{~ms})$ interstimulus intervals.

The data suggest that an important determinant of the shape of the H-reflex recovery curve is the frequency and extent of damping of oscillations in the system. The amplitude of the secondary facilitatory peak and the depth of the following trough would then be significantly affected by the amplitude and frequency of the underlying oscillations.

\section{References}

1 Hoffman P. Uber die Beziehungen der Sehnenreflexe zur willkürlichen Bewegung und zum Tonus. $Z$ Biolog 1918; 68:351-70.

2 Magladery JW, McDougal DB Jr. Electrophysiological studies of nerve and reflex activity in normal man, I Identification of certain reflexes in the electromyogram and the conduction velocity of peripheral nerve fibres. Bull Johns Hopkins Hosp 1950; 86:265-90.

3 Paillard J. Réflexes et régulations d'origine proprioceptive chez l'homme. Etude neurophysiologique et neuro-psychologique 1955; Paris: Arnette.

4 Diamantopoulos E, Zander Olsen P. Motoneurone excitability in normal subjects and patients with spasticity, rigidity, and cerebellar signs. Electroencephalogr Clin Neurophysiol 1965; 18:207-8.
5 Diamantopoulos E, Zander Olsen P. Excitability of motor neurones in spinal shock in man. $J$ Neurol Neurosurg Psychiatry 1967; 30:427-31.

6 Sax DS, Johnson TL, Cooper IS. Reflex recovery curves in extra-pyramidal disorders. Adv Neurol $1976 ; 14: 285-96$.

7 Lippold 0 . Oscillation in the stretch reflex arc and the origin of the rhythmical 8-12 c/s component of physiological tremor. J Physiol (London) 1970; 206:359-82.

8 Johnson SC. Hierarchical clustering schemes. Psychometrika 1967; 23:241-54.

9 Orguztöreli MN, Stein RB. An analysis of oscillations in neuro-muscular systems. J Math Biol 1975; 2:87-105.

10 Elble RJ, Randall JE. Motor-unit activity responsible for 8- to $12-\mathrm{Hz}$ component of human physiological finger tremor. J Neurophysiol 1976; 39(2):370-83.

11 Lemarre $Y$. Tremorgenic mechanism in primates. In: Meldrum BS, Marsden CD ed. Advances in Neurology, Vol 10, New York: Raven Press, 1975; 23-34.

12 Lee RG, Taffon WG. Motor responses to sudden limb displacements in primates with specific CNS lesions and in human patients with motor system disorders. Can J Neurol Sci 1975; 2:285-93.

13 Gassel MM. Critical review of evidence concerning long-loop reflexes excited by muscle afferents in man. J Neurol Neurosurg Psychiatry 1970; 33: 358-62.

14 Hayes KC, Robinson KL, Wood GA, Jennings LS. Assessment of the H-reflex excitability curve using a cubic spline function. Electroencephalog Clin Neurophysiol 1979; 46:114-7.

15 Katz R, Morin C, Pierrot-Deseilligny E, Hibino R. Conditioning of H-reflex by a preceding subthreshold tendon reflex stimulus. J Neurol Neurosurg Psychiatry 1977; 40:575-80. 